\title{
Renaissance of ion channel research and drug discovery by patch clamp automation
}

\begin{abstract}
"A mere 10 years ago, the automation of the delicate process of patch clamping was considered (nearly) impossible, and someone claiming such achievement would have been declared anywhere between pretentious and downright nuts by people skilled in the art, who possessed strong opinions on the matter."
\end{abstract}

Ion channels are highly intriguing biophysical entities that play an incredibly subtle role in the concerted actions in which they are involved, and that also have a crucial impact on inter- and intra-cellular communication. They respond to numerous kinds of stimuli and play a decisive role in the vitality of all living organisms. Ion channels are involved in the function of the cardiovascular and nervous systems and their malfunction underlies numerous diseases and indications. For exactly these reasons, ion channels have for decades been, and are still, the subject of in-depth research into a very broad range of important therapeutic areas. As membrane-bound proteins they are highly 'druggable' targets, being readily accessible to small molecules that are capable of fine tuning ion channel function by pharmacological modulation. Approximately $15 \%$ of the most successful drugs target ion channels [1], although ion channels have traditionally been difficult to screen due to a lack of adequate assays. Many of the marketed ion channel drugs were actually not discovered in rational drug-discovery programs, but rather empirically and by serendipity since the available ion channel-screening techniques typically confer a tradeoff between high content and high throughput.

Numerous different assay formats are compatible with the throughput requirements of primary screening $(100,000$ compounds or more), such as fluorescence assays based on ionor voltage-sensitive dyes, binding assays or ion flux measurements [2]. Although having been successfully employed and fulfilling a useful purpose, these assays suffer from a lack of voltage control, low resolution, low fidelity and a tendency to be prone to false-positive or falsenegative results. A further detailed assessment of screening technologies for ion channels can be found in this issue by Terstappen et al. [3] .
Approximately three decades ago, the pivotal development of the so-called patch clamp technique with giga-seal recordings of single ion channel action in real time by Hamill et al. opened new avenues for detailed biophysical characterization of ion channel behavior, their function, kinetics and pharmacology. Although it is the method of choice for ion channel studies and ubiquitous in academic research, the widespread employment of patch clamping within drug discovery and ionchannel screening has been limited due to the need for skilled operation, ultra-low throughput and highly expensive cost per data point.

A mere 10 years ago, the automation of the delicate process of patch clamping was considered (nearly) impossible, and someone claiming such achievement would have been declared anywhere between pretentious and downright nuts by people skilled in the art, who possessed strong opinions on the matter.

Nowadays, automated and parallel patch clamping is commonplace and a number of commercially available, and fairly mature, platforms have proven quite successful at fulfilling their tasks [4-9]. Most systems are based on planar chip substrates that contain one or more 1-2- $\mu \mathrm{m}$-sized apertures for capturing cells from suspension by means of suction; hence this approach is often referred to as 'planar patch clamping'. The cells are sealed to the substrate and either the whole cell configuration is reached by application of suction pulses, or pore-forming compounds are applied for perforated patch recordings allowing electrical access and control of the cellular membrane. The planar approach has two major benefits: automation and parallelization. As cells are positioned and sealed by suction, the recording configuration is reached automatically, thereby greatly 'deskilling' the process. By having an array of apertures in the chips, multiple cells can be recorded in parallel, permitting a major increase in data

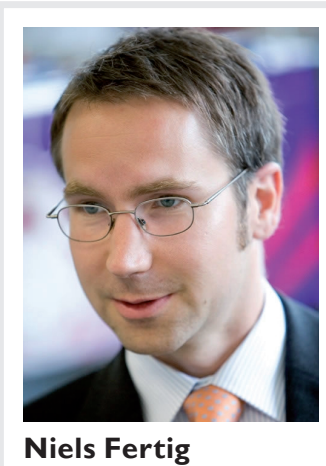

Author for correspondence: Nanion Technologies $\mathrm{GmbH}$ Erzgiessereistr 4, 80335, Munich, Germany

Tel.: +4989218997972

Fax: +4989218997960

E-mail: niels@nanion.de

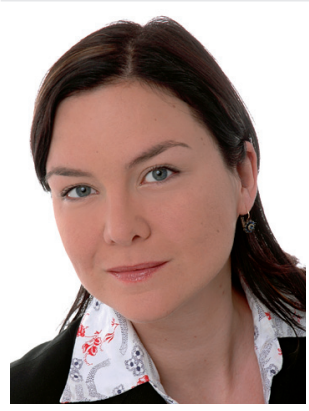

Cecilia Farre

Nanion Technologies GmbH, Erzgiessereistr 4, 80335, Munich, Germany 
throughput. The planar patch clamp technique overcomes some of the major limitations of the conventional patch clamp, while still offering the same advantages, such as temporal resolution, voltage control and direct measurement of a compound's mode of action.

Among the automated patch clamp platforms available on the market there are differences in performance, data quality and throughput.

'High data quality' refers to whether the platform is capable of generating recordings similar in data quality to that of conventional patch clamping. These platforms support properties such as 'giga-seal recordings', continuous voltage control of the membrane, recording during compound washout and compound washout, for example. Examples of such platforms are the PatchXpress (Molecular Devices), QPatch (Sophion, Biosciences), Port-a-Patch, Patchliner, SyncroPatch 96 (Nanion Technologies GmbH) and FlyScreen 8500 (Flyion GmbH).

The term giga-seal refers to the highly resistive interaction (giga-ohm) between the cell membrane and the planar patch clamp substrates. The giga-seal is a requirement for low-noise, high-quality recordings, with high temporal resolution. The throughput capability of platforms mentioned ranges from 50 to 7000 data points per day.

\section{"Automated patch clamp platforms offer higher throughput with maintained data quality, which means that a laboratory, or a core facility, can produce a large amount of high-quality data in less time."}

There are several platforms that possess lower data quality and, therefore, offer slightly higher data throughput. Here the old kid on the block is the IonWorks Quattro platform (Molecular Devices), recently joined by newcomers IonFlux (Fluxion Biosciences Inc) and Dynaflow HT (Cellectricon). The lower data quality outputs from these platforms arise from the poor electrical seal between the recording substrate and the cellular membrane (mega-ohm range). The poor electrical seal is compensated by subtracting the current leaking between the substrate and the membrane from the ionic currents originating from ion channel activity. Unfortunately, this lowers the resolution (i.e., increases the noise) and can have deleterious effects on voltage control of the cellular membrane, resulting in false negatives or positives, as well as narrowing the spectrum of applicable ion channels.
The mega-ohm platforms Dynaflow HT and IonFlux both have sophisticated fluidics, allowing drug washout, recordings during compound application as well as the collection of full dose responses from cells. The throughput from these products ranges between 3000 and 10,000 data points per day (figures provided by the companies), which remains to be proven by end users.

Another approach, the so-called 'population patch clamp' [10] is employed by the QPatchHTX, IonFlux ${ }^{\mathrm{TM}}$ and IonWorks ${ }^{\mathrm{TM}}$ Quattro platforms, where the average response from 10, 20 and 64 cells, respectively, is measured. The population patch clamp evens out the differences within the cell population, enabling measurements of smaller currents, and increases the success rates of the measurements to almost $100 \%$ (if the vendors are to be believed); however, it also suffers from the same drawback as with low-quality recordings (i.e., leak currents need to be compensated and if these change over time), meaning it is difficult or nearly impossible to distinguish compound action.

The drawbacks of mega-ohm and/or population patch clamping are that they impose limitations when, for example, investigating transient receptor channels (TRP), which are typically difficult to distinguish from leak or ion channels with rapid, voltage-dependent gating (e.g., in the case of sodium channels, in which currents are greatly affected by even minor drifts in the voltage control of the membrane). A further detailed assessment of screening technologies for ion channels can be found in this issue by Terstappen et al. [3].

Over the past 5 years the use of automated patch clamp systems has increased not only in drug discovery and contract research organization settings, but also within academic research. Industrial and academic requirements are similar, but also different.

One would think that the automated platforms would have difficulties in attracting a broad customer base within academic research due to the expense. This is true to some extent, however, the fact is that many of the mentioned platforms are actually being used in academic laboratories. Automated patch clamp platforms offer higher throughput with maintained data quality, which means that a laboratory, or a core facility, can produce a large amount of high-quality data in less time. This is important because, although it is not as important as in industry, time is money, even in a competitive academic environment, and every 
publication counts. Recent publications clearly show that academic researchers and science have benefited from planar patch clamping [11-14].

An important difference is, however, that an academic researcher has greater demands on experimental possibilities and experimental freedom than their industrial counterpart. There are platforms that are indeed useful for routine screening, but once the start button has been pressed no changes can be made to the protocol. Other automated patch clamp platforms offer a high level of flexibility and experimental freedom, allowing on-the-fly modifications of protocols, and unique experimental possibilities such as internal perfusion, temperature control, bilayer recordings and automated current clamp recordings. These platforms are also suitable for educational purposes, where students (in a matter of days) can obtain hands-on experience of high-quality patch clamp recordings with minimum training. The diversity of existing automated patch clamp platforms is good news for the end users, since the various needs of ion channel research and screening are covered.

Another difference is the 'quality', with regards to academic researchers. For most scientists megaohm seals and population patch clamping are just not good enough, often for good reasons: in academic settings the ion channels and cells studied are frequently quite challenging, for instance using primary cells, with small and/or uncharacterized currents and cells that need pampering to co-operate. Protocols are often more demanding, since the questions often require more than yes/no answers as to whether or not a compound alters current responses. Automated patch clamp in academic research can accelerate science and improve education - by introducing patch clamp technology to a larger audience. If we can get away from the fear of all the knobs, electronics and complicated data-acquisition systems often associated with conventional patch clamp electrophysiology, ion channel electrophysiology as a research field is bound for future growth.

Clearly, the planar patch clamp has been a 'facilitating technology', which significantly improved ion channel screening by increasing the throughput and ease-of-use of electrophysiology in drug discovery. Since automated patch clamp platforms became widely available, ion channels have seen a renaissance as therapeutic targets, which is reflected in the increased number of conferences dedicated to this area as well as the pharmaceutical industry's expanding ion channel programs.
However, the planar patch clamp platforms on the market today are far away from replacing the common work horses of primary ion channel screening. The data throughput offered by available platforms is still far too low.

A relevant question is, however, should automated patch clamp technology be used in primary screening? Or is the brute force approach to drug discovery, which is screening hundreds of thousands of compounds only ineffective, time consuming or even obsolete?

\section{"Since automated patch clamp platforms became widely available, ion channels have seen a renaissance as therapeutic targets, which is reflected in the increased number of conferences dedicated to this area as well as the pharmaceutical industry's expanding ion channel programs."}

When turning to the market for an answer, two things are currently required, indicating that the brute force approach is still considered valid. Throughput is being requested and, as a logical consequence, lower prices-per-datapoint are necessary. With these requirements, it is obvious that the work horses tend to miss out on potential drug candidates, because of their lack of sensitivity, and that they ideally should be replaced with high-quality systems such as planar patch clamps. Surprisingly enough, screeners seem very unwilling to pay more for more qualified data. As a response to the demand for a price-per-datapoint comparable with, for example, fluorescence methods, the planar patch clamp vendors, including ourselves, argue that a higher price-per-datapoint is justified if a drug can be found and evaluated faster, since rapid market entry and prolonged patent times for a drug is quantifiable by the day and would, in the long run, mean vast economic gain for the pharmaceutical companies.

So where will patch clamping be in 10 years' time? Looking into the crystal ball we believe it will be the same, but slightly different. Upscaling of patch clamping will still be the trend for the coming years, aimed at pushing the number of possible parallel recordings to its limits. The latest rumors tell about a platform capable of 384 simultaneous recordings; although, with the low-quality approach to patch clamping, it is fairly safe to assume or even predict that high-quality versions of this format are soon to follow. 
At the same time, after the moderate success of commercialized ion channel-active drugs, the use of recombinant cell lines and overexpressed ion channels in ion channel screening, drug companies are starting to look at more physiological cell systems, such as primary cells, or stem-cell derivates such as cardiomyocytes, neurons and hepatocytes for screening purposes.

\section{"We foresee that high-quality automated patch clamp platforms will be required to be capable of more than 'just' giga-seal recordings and reliable drug additions."}

These cells are, however, a lot more demanding in a number of ways. First of all, cell availability is lower. This means that the machines have to cope with a lower cell density, or more money has to be spent on the not so inexpensive stem cell lines, or more animals have to be used for primary cell preparations, which also raises ethical considerations.

Second, ion channel expression is typically much lower in the cell types mentioned, compared with using over-expressing cell lines, which, in turn, require highly resolved measurements of the smaller, and often more complex currents. Here the high-quality screening systems clearly have the upper hand in terms of giving highly qualified information about ion channel and compound action.

Third, the 'easy' ion channel targets are long gone. That means that more demanding targets such as TRP channels, including the thermoTRPs, need adequate patch clamp measurement methods for correct evaluation - such as temperature activation and careful use of leak subtraction methods (if any).

Further on, the ensemble effect of ion channels is important, for example, when looking at firing behavior in neurons. Here, automated current clamp measurements would be the method of choice, revealing how a drug affects the action potentials rather than individual ion channels. This aspect is also important in the safety testing of drugs, since some compounds had effect on cardiac channels such as hERG in vitro (e.g., Verapamil) but exhibited no cardiac safety issues in vivo [15]. The action potential recordings give a better indication of a compound's potential safety liability issues, saving time, money and worry in the preclinical testing phase as well as reducing the risk of discarding good (and costly) compounds due to a lack of knowledge.
It is definitively safe to state that automated ion channel platforms have earned their place within industry and academic research.

If it were technically possible, most people would probably agree that substituting the primary screening work horses for automated patch clamp would give completely new, and also more qualified, leads and drastically reduce the danger of missing the gold nuggets in the 100,000 plus compound libraries. But is it realistic? Yes, we think it is possible - but new technologies and strategies are required. Automated patch clamp definitively has the capability to resolve small modifications in ion channel kinetics and action, in ways that fluorescence, flux and affinity assays do not. Throughput is still the bottleneck today, a throughput of 5000-10,000 datapoints per day is achievable. Given that the platforms are pushed towards higher parallelism, and thus higher throughput, automated patch clamp could be used earlier in the drug-discovery process.

Another option that would be viable today, even for a 100,000 compound screen, is to apply multiple compounds to cells, either sequentially or at once, with a well-considered deconvoluting strategy. However, here, data analysis and, later, data mining, will be just as important for a successful outcome.

With more challenging targets and, consequently, more demanding cellular assays an even more rational approach to ion channel drug screening needs to be taken. Options for the future might be computer simulations and 'virtual screening' before actual patch clamp-based screening or drug safety testing.

\section{Future perspective}

To conclude, ion channel screening will be more diversified than it is now, requiring the usual themes: higher throughput, lower consumable cost, but also higher levels of experimental flexibility. We foresee that high-quality automated patch clamp platforms will be required to be capable of more than 'just' giga-seal recordings and reliable drug additions, such as temperature control, action potential recordings and bilayer recordings, for example, to encompass the diversified requirements of future ion channel screening. Despite the current changes and challenges that drastically affect the pharmaceutical industry, ion channel screening is here to stay with an ever-expanding role. Ion channel screening of tomorrow will be similar to what we see today, yet also different based on the ever-changing customer needs. 


\section{Financial \& competing interests disclosure}

The authors are employees of Nanion Technologies, a company that is involved in the provision of automated patch clamp instrumentation. The authors have no other relevant affiliations or financial involvement with any organization or entity with a financial interest in or financial conflict with the subject matter or materials discussed in the manuscript. This includes employment, consultancies, honoraria, stock ownership or options, expert testimony, grants or patents received or pending, or royalties.

No writing assistance was utilized in the production of this manuscript.

\section{Bibliography}

1 Overington JP, Al-Lazikani B, Hopkins AL. How many drug targets are there? Nat. Rev. Drug Discov. 5, 993-996 (2006).

2 Gonzalez JE, Oades K, Leychkis Y et al. Cell-based assays and instrumentation for screening ion channel targets. Drug Discov. Today. 4, 431-439 (1999).

3 Terstappen GC, Roncarati R, Dunlop J et al. Screening technologies for ion channel drug discovery. Future Med. Chem. 2(5), 715-730 (2010).

4 Hamill OP, Marty A, Neher E et al. Improved patch-clamp techniques for high-resolution current recording from cells and cell-free membrane patches. Pflugers Arch. 391(2) 85-100 (1981)

5 Dunlop J, Bowlby M, Peri R et al. Highthroughput electrophysiology: an emerging paradigm for ion-channel screening and physiology. Nature Rev. Drug Disc. 7 , 358-368 (2008).

6 Mathes C. QPatch: the past, present and future of automated patch clamp. Expert Opin. Ther. Targets 10, 319-327 (2006).
7 Farre C, Stoelze S, Haarmann C et al. Automated ion channel screening: patch clamping made easy. Expert Opin. Ther. Targets. 11, 557-565 (2007).

8 Farre C, Haythornthwaite A, Haarmann C et al. Port-a-Patch and Patchliner: high fidelity electrophysiology for secondary screening and safety pharmacology. Comb. Chem. HTS 12 (1), 24-37 (2009).

9 Behrends JC, Fertig N. Planar patch clamping. In: Neuromethods Patch Clamp Analysis Advanced Techniques (Volume 38). Walz W (Ed.). Humana Press, Totowa, Canada, 411-433 (2007).

10 Kiss L, Bennett PB, Uebele VN et al. High-throughput ion-channel pharmacology: planar-array-based voltage clamp. Assay Drug Dev. Technol. 1,127-135 (2003).

11 Schroeder K, Neagle B, Trezise DJ et al. Ionworks HT: a new high-throughput electrophysiology measurement platform. J. Biomol. Screen. 8, 1-14 (2003).

12 Finkel A, Wittel A, Yang N et al. Population patch clamp improves data consistency and success rates in the measurement of ionic currents. J. Biomol. Screen. 11, 488-496 (2006).
13 Xu SZ, Sukumar P, Zeng F et al. TRPC channel activation by extracellular thioredoxin. Nature 451(7174), 69-72 (2008).

14 Milligan CJ, Li J, Sukumar P et al. Robotic multiwall planar patch-clamp for native and primary mammalian cells. Nature Protocols 4(2), 244-255 (2009).

15 Kahlig KM, Saridey SK, Kaja A et al. Multiplexed transposon-mediated stable gene transfer in human cells. Proc. Natl Acad. Sci. USA 107(4), 1343-1348 (2010).

16 Kreir M, Farre C, Beckler M et al. Rapid screening of membrane protein activity: electrophysiological analysis of $\mathrm{OmpF}$ reconstituted in proteoliposomes. Lab-on- $a-$ Chip. 4, 587-595 (2008).

17 Fenichel RR, Malik M, Antzelevitch C et al. Drug induced torsade de pointes and implications for drug development. J. Cardiovasc. Electrophysiol. 15(4), 475-495 (2004). 